around the LCA orifice (Fig. 2, middle). This atheromatous material was removed very carefully, creating a wide orifice. Then a saphenous vein patch $(15 \times 10 \mathrm{~mm})$ was sutured with 7-0 Prolene sutures from the inferior border of the LMT incision up to the junction of the aortic wall, terminating $5 \mathrm{~mm}$ below the end of the aortotomy. After the saphenous vein patch was secured, the entire course of the LMT was carefully examined. The transected aorta was reconstructed with a continuous 4-0 Prolene suture (Fig. 2, bottom).

The patient was weaned from the pump without difficulty and transferred to the intensive care unit in good condition. There were no postoperative changes in the electrocardiogram. The recovery was uneventful, and the patient was discharged in good condition. Postoperative catheterization revealed a large LCA orifice and LMT (Fig. 1).

Surgical angioplasty of the LMT provides physiologic coronary perfusion and good late results without the difficulties associated with graft occlusion. ${ }^{4}$ Impaired LMT flow, however, may result in significant damage to the myocardium. ${ }^{5}$ Physiologic reconstruction after exposure of the entire LMT is necessary to provide the best flow through this vessel. The "superior approach" de- scribed here is an effective way to gain wide exposure of the LMT for surgical manipulation.

\section{REFERENCES}

1. Dion R, Verhelst R, Matta A, Rousseau M, Goenen M, Chalant C. Surgical angioplasty of the left main coronary artery. J Thorac Cardiovasc Surg 1990;99:241-50.

2. Hitchcock JF, Robles de Medina EO, Jambroes G. Angioplasty of the left main coronary artery for isolated left main coronary artery disease. J Thorac Cardiovasc Surg 1983;85: 880-4.

3. Sabiston DC, Ebert PA, Friesinger GC, et al. Proximal endarterectomy: arterial reconstruction for coronary occlusion at the aortic origin. Arch Surg 1965;91:758-64.

4. Sen RC, Hitter E, Ranquin R, Cawwelaert V, Lieber S, Vanden-Branden F. Surgical coronary angioplasty for left main vasospasm. Am Heart J 1995;129:399-400.

5. Favaloro RG, Effler DB, Groves LK, Sheldon WC, Shirey EK Sones FM. Severe segmental obstruction of the left main coronary and its division: surgical treatment by the saphenous vein graft technique. J Thorac Cardiovasc Surg 1970;60:46982

\title{
WHOLE BLOOD HEPARIN CONCENTRATION MEASUREMENTS BY AUTOMATED PROTAMINE TITRATION AGREE WITH PLASMA ANTI-XA MEASUREMENTS
}

\author{
G. J. Despotis, MD, J. H. Joist, MD, PhD, L. T. Goodnough, MD, S. A. Santoro, MD, PhD, and E. Spitznagel, PhD, \\ St. Louis, Mo.
}

Findings recently reported by our group indicated that point-of-care monitoring of heparin concentration by an automated protamine sulfate titration (PST) method (Hepcon HMS, Medtronic Hemotec, Inc., Englewood, Colo.) can reduce excessive microvascular bleeding and blood component transfusion. ${ }^{1}$ That study was preceded by another evaluation in which we found a high degree of correlation between heparin concentration determined by

From the Departments of Anesthesiology, Internal Medicine, Pathology, and Surgery, Washington University School of Medicine, St. Louis, Mo., and the Departments of Internal Medicine and Pathology, St. Louis University School of Medicine, St. Louis, Mo.

Received for publication Sept. 23, 1996; accepted for publication Oct. 22, 1996.

Address for reprints: George Despotis, MD, Division of Cardiothoracic Anesthesiology, Department of Anesthesiology, Box 8054, Washington University School of Medicine, 660 South Euclid Ave., St. Louis, MO 63110.

J Thorac Cardiovase Surg 1997;113:611-3

Copyright () 1997 by Mosby-Year Book, Inc.

$0022-5223 / 97 \$ 5.00+0 \quad \mathbf{1 2 / 5 4 / 7 8 7 6 8}$ plasma anti-Xa and whole blood automated PST. ${ }^{2}$ Hardy and colleagues ${ }^{3}$ recently published a comparison of heparin measurements by Hepcon automated PST and a laboratory-based anti-Xa chromogenic assay in a relatively small number of patients undergoing cardiac operation with the use of cardiopulmonary bypass (CPB). In contrast to our observations of a strong linear relationship between whole blood PST and plasma anti-Xa measurements, ${ }^{2}$ these investigators found unacceptably high variability between the two heparin concentration measurements. In explaining this discrepancy, Hardy and colleagues ${ }^{3}$ suggested that our data analysis was limited and potentially misleading because we did not use bias analysis. We therefore reanalyzed our data with a bias analysis method as used by that group.

Plasma anti-Xa and whole blood PST measurements (duplicate) on blood specimens obtained at sequential operative times ( 4 to 6 per patient) from 62 patients undergoing cardiac operations were reanalyzed. ${ }^{2}$ Whole blood (WB) PST heparin values were converted to plasma equivalent values (PE) using concurrently measured hematocrit (Hct) values with the following formula: PE WB PST $=($ WB PST $\times 100 /[100-\mathrm{Hct}])$. The mean difference or bias between plasma anti-Xa 


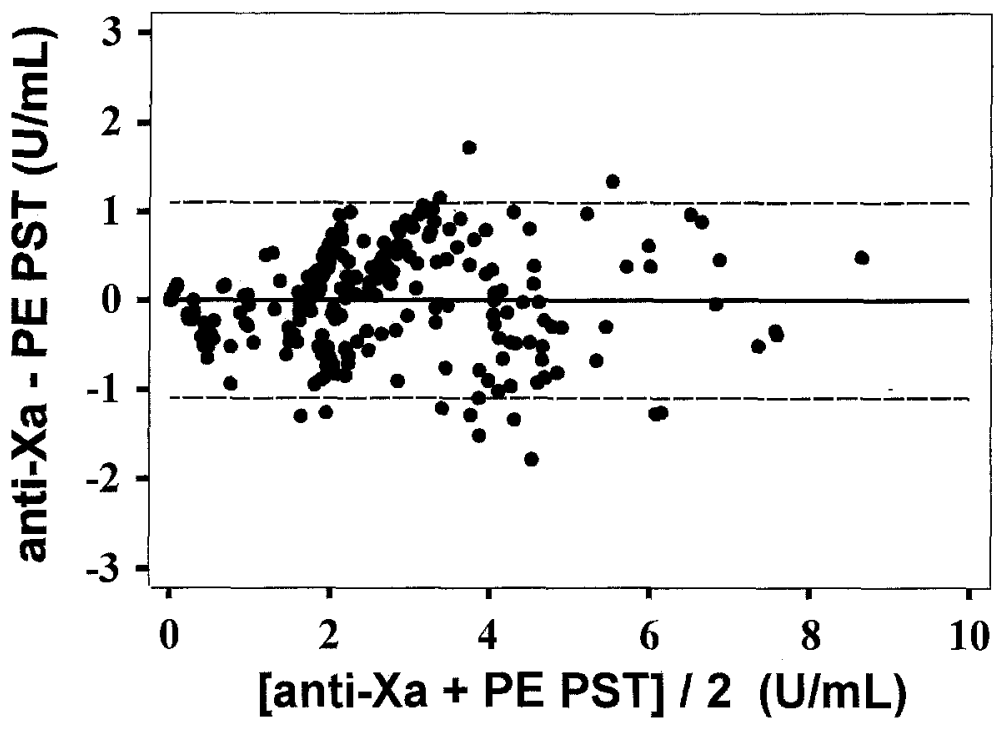

Fig. 1. Bias analysis of plasma anti-Xa versus plasma-equivalent whole blood heparin concentration measurements. Heparin concentration in plasma was measured with use of an anti-Xa chromogenic assay and whole blood heparin concentration values were measured with an automated PST method with use of the Hepcon instrument. Whole blood heparin concentration values were converted to plasma equivalent values $(P E)$ with the following formula: PE PST $=($ PST $\times 100 /[100-$ hematocrit $])$. The differences between anti-Xa and whole blood heparin concentration measurements are plotted on the $\mathrm{y}$ axis and the averages of the two heparin concentration values are plotted on the $\mathrm{x}$ axis. The mean (bias) and plus or minus two standard deviations of the differences are represented by the horizontal lines. $n=310$.

and corrected whole blood PST measurements was $0.002 \pm 0.53 \mathrm{U} / \mathrm{ml}$. Fig. 1 shows the results of the bias analysis with means and two standard deviation confidence intervals for the 310 measurement pairs. The mean difference obtained using duplicate measurements was similar to those obtained with each of the two individual measurements (measurement 1:0.008 \pm 0.56 ; measurement $2:-0.004 \pm 0.56$ ) .

The findings of this further analysis of our previously published data indicate that measurements of heparin concentration derived from the Hepcon HMS automated protamine titration method agree well with laboratory-based anti-Xa measurements of heparin concentration when the sensitivity limit of $0.7 \mathrm{U} / \mathrm{ml}$ for the Hepcon HMS method is applied as suggested by Hardy and colleagues. ${ }^{3}$ Several factors may account for the discrepancy between our findings and those of Hardy and colleagues..$^{3}$ Most important, in their study, hematocrit values used for the conversion of whole blood heparin concentration into plasma equivalent values were obtained from arterial blood gas analysis determined at 30-minute intervals during CPB and not from a conventional hemocytometer with blood specimens obtained simultaneously with those used for measurement of heparin concentration (as was done in our study), and this may have led to miscalculation of plasma-equivalent whole blood heparin concentrations as acknowledged by the authors. ${ }^{3}$ Differences in standardization and performance of laboratory-based anti-Xa chromogenic assays may have also contributed to the different results obtained by Hardy and colleagues. ${ }^{3}$ The wide variability in anti-Xa measurement values between various institutions with low-molecularweight heparin ${ }^{4}$ or the inconsistency between laboratory-based anti-Xa and protamine titration methods, ${ }^{5}$ or both of these reasons, may also in part account for the discrepancy between our studies.

Maintenance of adequate, patient-specific heparin concentrations with the use of the Hepcon instrument has been shown to reduce excessive hemostatic system activation and preserve coagulation factors during $\mathrm{CPB},{ }^{6}$ which may in part account for the significant reduction in blood component transfusion requirements seen with this approach. ${ }^{1}$ In an attempt to explain the benefits found in our previous studies, Hardy and colleagues ${ }^{3}$ suggested that administration of higher heparin and lower protamine doses may have accounted for the improved outcomes. Although these findings may be the result of administration of greater overall heparin doses, the relative importance of administering heparin on the basis of patientspecific requirements cannot be overlooked. In our previous evaluation, ${ }^{1}$ the total heparin dose was directly related to the concentration of heparin maintained during CPB that was designated for each patient on the basis of 
the patient's anticoagulant response to heparin before $\mathrm{CPB}$ by use of a standard assay (activated clotting time). Finally, we have recently evaluated the use of continuous infusions of heparin during $\mathrm{CPB}$ to maintain stable, adequate heparin concentrations. Unpublished data from this study confirm the reliability and usefulness of on-site, whole blood heparin concentration measurements by the Hepcon HMS system.

\section{REFERENCES}

1. Despotis GJ, Joist JH, Hogue CW, et al. The impact of heparin concentration and activated clotting time monitoring on blood conservation: a prospective, randomized evaluation in patients undergoing cardiac operations. J Thorac Cardiovasc Surg 1995;110:46-54.

2. Despotis GJ, Summerfield AL, Joist JH, et al. Comparison of activated coagulation time and whole blood heparin measure- ments with laboratory plasma anti-Xa heparin concentration in patients having cardiac operations. $\mathrm{J}$ Thorac Cardiovasc Surg 1994;108:1076-82.

3. Hardy JF, Belisle S, Robitaille D, Perrault J, Roy M, Gragnon L. Measurement of heparin concentration in whole blood with the Hepcon/HMS device does not agree with laboratory determination of plasma heparin concentration using a chromogenic substrate for activated factor X. J Thorac Cardiovasc Surg 1996;112:154-61.

4. Barrowcliffe TW, Curtis AD, Tomlinson TP, Hubbard AR, Johnson EA, Thomas DP. Standardization of low molecular weight heparins: a collaborative study. Thromb Haemost 1985;54:675-9.

5. Hirsh J. Heparin [Review]. N Engl J Med 1991;324:1565-74.

6. Despotis GJ, Joist JH, Hogue CW, et al. The effect of higher heparin concentrations on preservation of hemostasis in cardiac surgical patients. Thromb Haemost 1996;76:902-8. 